\title{
AN INFINITE VERSION OF THE PÓLYA ENUMERATION THEOREM
}

\author{
ROBERT A. BEKES \\ Mathematics Department, Santa Clara University \\ Santa Clara, CA 95053 \\ (Received July 29, 1991 and in revised form December 17, 1991)
}

\begin{abstract}
Using measure theory, the orbit counting form of Pólya's enumeration theorem is extended to countably infinite discrete groups.
\end{abstract}

KEY WORDS AND PHRASES. Infinite discrete group, Pólya's emumeration theorem . 1991 AMS SUBJECT CLASSIFICATION CODE. 05A15

\section{INTRODUCTION.}

Let $G$ be countable discrete group acting as permutations on a countable set $D$. Let $S$ be a finite set with cardinality, $|S|=N$. Denote by $S^{D}$ the set of functions from $D$ to $S$. For $\gamma \in S^{D}$ define $g \gamma \in S^{D}$ by $g \gamma(d)=\gamma\left(g^{-1} d\right)$. For a subgroup $K$ of $G$ let $\Delta_{K}$ be a set of representatives for the orbits of $K$ in $S^{D}$. Let 36 be a Hilbert space with orthonormal basis $\left\{e_{\gamma}: \gamma \in S^{D}\right\}$ and inner product $<,>$. Define a unitary representation of $G$ on 36 by $\pi(g) e_{\gamma}=e_{g \gamma}$.

The number of orbits of $G$ in $S^{D}$ is denoted by $\left|\Delta_{G}\right|$. For finite $G$ and $D$ this can be counted by the Pólya enumeration theorem. Specifically, for each $g \in G$, let $c_{i}(g)$ be the number of cycles of length $i$ in the representation of $g$ as a product of disjoint cycles in $D$ and let $M(g)=y_{1}{ }^{c_{1}(g)} \cdots y_{n}{ }^{{ }(g)}$, where $n=|D|$. The cycle index of $G$ on $D$ is the polynomial $P_{G}=$ $\frac{1}{|G|} \sum_{g \in G} M(g)$. Denote by $\sigma P_{G}$ the value $P_{G}$ at $y_{\mathrm{t}}=N, i=1$ to $n$. Pólya's enumeration theorem, see Pólya [1], says that $\left|\Delta_{G}\right|=\sigma P_{G}$.

Define the operator $T_{G}$ on $\mathfrak{H}_{6}$ by $T_{G}=\frac{1}{|G|} \sum_{g \in G} \pi(g)$. Then it can also be shown, see Williamson [2], that $\left|\Delta_{G}\right|=\operatorname{trace}\left(T_{G}\right.$ on $\left.{ }_{6}\right)$. It is these two ways of measuring a set of representatives for orbits that we extend to infinite $G$ and $D$.

\section{THE MAIN RESULTS.}

If we view $S$ as a finite group with the discrete topology, then $S^{D}$ is a compact group in the product topology. Let $\mu$ be normalized Haar measure on $\mathrm{S}^{D}$.

For $g \in G$ and $\gamma \in S^{D}$ define $f(\gamma)=<\pi(g) e_{\gamma}, e_{\gamma}>$. Then $f(\gamma)= \begin{cases}1 & \text { if } g \gamma=\gamma \\ 0 & \text { otherwise }\end{cases}$

LEMMA 1. $\mathrm{f}$ is measurable.

PROOF. Let $f_{i}\left(\gamma\left(d_{1}\right)\right)=\left\{\begin{array}{ll}1 & \text { if } \gamma\left(g^{-1} d_{1}\right)=\gamma\left(d_{1}\right) \\ 0 & \text { otherwise }\end{array} \quad\right.$ and $h_{n}(\gamma)=\prod_{i=1}^{n} f_{i}\left(\gamma\left(d_{v}\right)\right)$.

Then $h_{n}$ is measurable for all $n$. Now $g \gamma=\gamma$ if and only if $\gamma$ is constant on the orbits of $g$. But this happens if and only if $\gamma\left(g^{-1} d\right)=\gamma(d)$ for all $d \in D$. Therefore $f(\gamma)=1$ if and only if $f_{\mathrm{t}}\left(\gamma\left(d_{2}\right)\right)=1$ 
for all $i$. This shows that $f(\gamma)=\lim _{n \rightarrow \infty} h_{n}(\gamma)$ and therefore measurable by Hewett and Stromberg $[3,22.24 \mathrm{~b}]$.

We write $D=\left\{d_{1}, d_{2}, d_{3}, \ldots\right\}$ and let $D_{n}=\left\{d_{1} \ldots, d_{n}\right\}$. Let $\langle g\rangle$ be the subgroup generated by $g$ and $\langle g\rangle d$ the orbit of $d$ under $\langle g\rangle$. For each $n$ and each $k \leq n$ let $c_{k}{ }^{n}(g)$ be the number of distinct cycles of $g$ such that $\left|\langle g\rangle d \cap D_{n}\right|=k$. Form the monomial $M^{n}(g)=\frac{1}{N^{n}} y_{1}{ }^{c_{1}{ }^{n}(g)} y_{2}{ }^{c_{2}{ }^{n}(g)} \ldots y_{n}{ }^{c_{n}{ }^{n}(g)}$.

LEMMA 2. $\int_{S^{D}}<\pi(g) e_{\gamma}, e_{\gamma}>d \mu(\gamma)=\lim _{n \rightarrow \infty} \sigma M^{n}(g)$.

PROOF. From the proof of Lemma 1 we saw that $\left\langle\pi(g) e_{\gamma}, e_{\gamma}\right\rangle=\lim _{n \rightarrow \infty} h_{n}(\gamma)$. So by the dominated convergence theorem, $\int_{S^{D}}\left\langle\pi(g) e_{\gamma}, e_{\gamma}\right\rangle d \mu(\gamma)=\lim _{n \rightarrow \infty} \int_{S^{D}} h_{n}(\gamma) d \mu(\gamma)$. But now $h_{n}(\gamma)=1$

if and only if $\gamma$ is constant on the intersection of the orbits of $g$ with $D_{n}$ otherwise $h_{n}(\gamma)=0$. Let $B_{n}=\left\{\gamma: \gamma\right.$ is constant on the intersection of the orbits of $g$ with $\left.D_{n}\right\}$. Then $\int_{S^{D}} h_{n}(\gamma) d \mu(\gamma)=$ $\mu\left(B_{n}\right)$. Since there are $N$ choices for the value of $\gamma$ on each orbit meeting $D_{n}$ and no restrictions on $\gamma$ outside $D_{n}$, we get $\mu\left(B_{n}\right)=\frac{1}{N^{n}} N^{c_{1}{ }^{n}(g)} \ldots N^{c_{n}{ }^{n}(g)}=\sigma M^{n}(g)$.

Let $G_{o}$ be the subgroup of $G$ consisting of all those $g \in G$ having only a finite number of cycles in $D$ of length greater than 1 .

LEMMA 3. $\int_{S^{D}}<\pi(g) e_{\gamma}, e_{\gamma}>d \mu(\gamma)=0$ for all $g \notin G_{o}$.

PROOF. Suppose $g \notin G_{o}$. Then there either exists $k_{o}$ such that $c_{k_{o}}{ }^{n}(g) \rightarrow \infty$ as $n \rightarrow \infty$ or there exists an increasing sequence $\left\{k_{n}\right\}$ such that $c_{k_{n}}{ }^{n}(g) \geq 1$. In the first case, for $n \geq k_{o}$, $n-\sum_{i=1}^{n} c_{i}{ }^{n}(g)=\sum_{i=1}^{n}(i-1) c_{i}{ }^{n}(g) \leq c_{k_{0}}{ }^{n}(g)$. So with $B_{n}$ as in the proof of Lemma 2, we get $0 \leq \int_{S^{D}}^{i=1}\left\langle x(g) e_{\gamma}, e_{\gamma}>d \mu(\gamma)=\lim _{n \rightarrow \infty} \mu\left(B_{n}\right) \leq \lim _{n \rightarrow \infty} N^{-c_{k_{0}}{ }^{n}(g)}=0\right.$. In the second case we get $n-\sum_{i=1}^{n} c_{i}{ }^{n}(g) \leq k_{n}-1$ and so $0 \leq \int_{S^{D}}<\pi(g) e_{\gamma}, e_{\gamma}>d \mu(\gamma)=\lim _{n \rightarrow \infty} \mu\left(B_{n}\right) \leq \lim _{n \rightarrow \infty} N^{-\left(k_{n}-1\right)}=0$.

For each $k$ let $F_{k}=\left\{g \in G: g d_{i}=d_{i}\right.$ for all $\left.i>k\right\}$. Then $\left\{F_{k}\right\}$ is a nondecreasing sequence of subgroups with $\bigcup_{k=1}^{\infty} F_{k}=G_{0}$. Suppose $G=\left\{g_{1}, g_{2}, \ldots\right\}$ and let $G_{m}=\left\{g_{1}, \ldots, g_{m}\right\}$. Assume $G$ is ordered in such a way that there exists a subsequence $\left\{m_{k}\right\}$ with $G_{o} \cap G_{m_{k}}=F_{k}$.

Let $F$ be a finite subset of $\mathrm{G}$. Define the $n^{\text {th }}$ cycle index of $F$ to be the polynomial $P_{F}^{n}=$ $\frac{1}{|F|} \sum_{g \in F} M^{n}(g)$. Define the operator $T_{F}$ on 36 by $T_{F}=\frac{1}{|F|} \sum_{g \in F} \pi(g)$. Write $P_{m}{ }^{n}$ for $P_{G_{m}}{ }^{n}$ and $T_{m}$ for $T_{G_{m}}$. 
THEOREM 4. $\Delta_{G_{0}}$ is closed and

$\mu\left(\Delta_{G_{o}}\right)=\lim _{k \rightarrow \infty}\left\{\frac{m_{k}}{\left|G_{m_{k}} \cap G_{o}\right|} \lim _{n \rightarrow \infty} \sigma P_{m_{k}}{ }^{n}\right\}=\lim _{k \rightarrow \infty} \frac{m_{k}}{\left|G_{m_{k}} \cap G_{o}\right|} \int_{S^{D}}<T_{m_{k}} e_{\gamma}, e_{\gamma}>d \mu(\gamma)$.

PROOF. Fix $k$ and let $D_{k^{\prime}}=\left\{d_{k+1}, d_{k+2} \ldots\right\}$. If $\alpha_{1}, \ldots, \alpha_{s}$ are representatives for the orbits of $F_{k}$ in $S^{D_{k}}$, then $\Delta_{F_{k}}=\left\{\alpha_{1}, \ldots, \alpha_{s}\right\} \times S^{D_{k^{\prime}}}$. Therefore $\Delta_{F_{k}}$ is closed and $\mu\left(\Delta_{F_{k}}\right)=\frac{s}{N^{k}}$. Let $\mathcal{J}_{k}$ be a Hilbert space with orthonormal basis $\left\{e_{\alpha}: \alpha \in S^{D_{k}}\right\}$. By Williamson [2], $s=\operatorname{trace}\left(T_{F_{k}}\right.$ on $\left.{ }^{3} 6_{k}\right)=$ $\sigma P_{F_{k}}$, where $P_{F_{k}}$ is the usual cycle index of $F_{k}$ on $D_{k}$. Note that $\sigma P_{F_{k}}=N^{k} P_{F_{k}}{ }^{n}$ for all $n \geq k$. By Lemma 3, $\frac{m_{k}}{\left|G_{m_{k}} \cap G_{o}\right|} \lim _{n \rightarrow \infty} \sigma P_{m_{k}}{ }^{n}=\lim _{n \rightarrow \infty} \sigma P_{F_{k}}{ }^{n}$. Therefore $\frac{m_{k}}{\left|G_{m_{k}} \cap G_{o}\right|} \lim _{n \rightarrow \infty} \sigma P_{m_{k}}{ }^{n}=\frac{1}{N^{k}} \sigma P_{F_{k}}$. By Lemma 2, $\quad \lim _{n \rightarrow \infty} \sigma P_{m_{k}}{ }^{n}=\int_{S^{D}}<T_{m_{k}} e_{\gamma}, e_{\gamma}>d \mu(\gamma)$. So we get $\mu\left(\Delta_{F_{k}}\right)=\frac{m_{k}}{\left|G_{m_{k}} \cap G_{o}\right|} \lim _{n \rightarrow \infty} \sigma P_{m_{k}}{ }^{n}=$ $\frac{m_{k}}{\left|G_{m_{k}} \cap G_{o}\right|} \int_{S^{I}}<T_{m_{k}} e_{\gamma}, e_{\gamma}>d \mu(\gamma)$.

Since $F_{k} \subseteq G_{o}$ we can assume that $\Delta_{G_{o}} \subseteq \Delta_{F_{k}}$ for all $k$. Therefore $\Delta_{G_{o}} \subseteq \bigcap_{k=1}^{\infty} \Delta_{F_{k}}$. We claim that $\Delta_{G_{o}}=\bigcap_{k=1}^{\infty} \Delta_{F_{k}}$. To see this suppose that $\gamma \in \Delta_{F_{k}}$ for all $k$. Then there exists $\gamma, \in \Delta_{G_{o}}$ and $g \in G_{o}$ such that $\gamma=g \gamma$. Since $G_{o}=\bigcup_{k=1}^{\infty} F_{k}$ there exists $k_{o}$ such that $g \in F_{k_{o}}$. Therefore $\gamma$ and $\gamma$, represent the same orbit of $F_{k_{o}}$ in $S^{D}$. Since $\gamma$ and $\gamma \prime \in \Delta_{F_{k_{o}}}$ we get $\gamma=\gamma$. This proves the claim.

It follows that $\Delta_{G_{o}}$ is closed and hence measurable. Therefore $\mu\left(\Delta_{G_{o}}\right)=\lim _{k \rightarrow \infty} \mu\left(\Delta_{F_{k}}\right)$. This completes the proof of the theorem.

Suppose now that $G$ is in no particular order. We show how to compute $\mu\left(\Delta_{G_{o}}\right)$. Let $A_{m}$ $=G_{m} \cap G_{0}$ and let $T_{A_{m^{\prime},}}=\left(T_{A_{m}}\right)^{n}$.

THEOREM 5. $\quad \mu\left(\Delta_{G_{o}}\right)=\lim _{m \rightarrow \infty} \lim _{n \rightarrow \infty} \int_{S^{D}}<T_{A_{m^{\prime}},} e_{\gamma}, e_{\gamma}>d \mu(\gamma)$.

PROOF. Exists $m_{o}$ so that $1 \in G_{m_{o}}$. Fix $m \geq m_{o}$ and let $H_{m}$ be the subgroup of $G_{o}$ generated by $A_{m}$. Define a probability measure $\nu$ on $H_{m}$ by $\nu(g)=\frac{1}{\left|A_{m}\right|}$ if $g \in A_{m}$ and $\nu(g)=0$ otherwise. Let $\nu^{* n}$ be the $\mathrm{n}$-fold convolution of $\nu$ with itself and $U$ the uniform probability measure on $H_{m}$. Then by Diaconis [4, pg23], $\left\|\nu^{* n}-U\right\| \rightarrow 0$ where $\|\cdot\|$ is the total variation norm. If we extend the representation $\pi$, in the usual way, to the set of measures on $H_{m}$ we get $\pi\left(\nu^{* n}\right)=\left(T_{A_{m}}\right)^{n}=T_{A_{m},{ }^{n}}$ and $\pi(U)=T_{H_{m}}$. It follows, therefore, that $\lim _{n \rightarrow \infty}\left\langle T_{A_{m}, n} e_{\gamma}, e_{\gamma}\right\rangle=\left\langle T_{H_{m}} e_{\gamma}, e_{\gamma}\right\rangle$ for all $\gamma \in S^{D}$. By the dominated convergence theorem, $\lim _{n \rightarrow \infty} \int_{S^{D}}<T_{A_{m}, n} e_{\gamma}, e_{\gamma}>d \mu(\gamma)=\int_{S^{D}}<T_{I_{m}} e_{\gamma}, e_{\gamma}>d_{\mu}(\gamma)$. Then as in the proof of Theorem 4, we get $\left.\mu\left(\Delta_{H_{m}}\right)=\int_{S^{D}}<T_{H_{m}} e_{\gamma}, e_{\gamma}\right\rangle d \mu(\gamma)$. The result follows since $G_{o}=\bigcup_{m=1}^{\infty} H_{m}$. 


\section{EXAMPLE.}

Suppose $D=\bigcup_{n=1}^{\infty} D_{n}$, where the $D_{n}$ are disjoint and finite and that $G$ sends $D_{n}$ into itself. Then if $G_{n}$ is $G_{\text {restricted to }}^{n=1} D_{n}, G$ is isomorphic to the product $\prod_{n=1}^{\infty} G_{n}$. In this case the product measure $\mu$ on $S^{D}$ need no longer come from uniform measures on $S$.

Let $S=\left\{s_{1}, \cdots, s_{k}\right\}$ and let the measure $\nu$ on $S$ be defined by $\nu\left(s_{i}\right)=a_{i}$. If $\left|D_{n}\right|=m_{n}$ define the measure $\mu_{n}$ on $S^{D_{n}}$ by $\mu_{n}=\prod_{i=1}^{m_{n}} \nu$. Let $\Delta_{n}$ be representatives for the orbits of $G_{n}$ in $S^{D_{n}}$ and $P_{G_{n}}$ the cycle index. Then using the pattern inventory from Pólya's enumeration theorem, see Pólya and Read [1], we get $\mu_{n}\left(\Delta_{n}\right)=P_{G_{n}}\left(\sum_{i=1}^{k} a_{i}, \sum_{i=1}^{k} a_{i}{ }^{2}, \cdots, \sum_{i=1}^{k} a_{i}{ }^{n}\right)$. Let $\mu=\prod_{n=1}^{\infty} \mu_{n}$ and let $\Delta$ be representatives for the orbits of $G$ in $R^{D}$. Then, as in the proof of Theorem 4, we get that $\mu(\Delta)=\lim _{n \rightarrow \infty} \prod_{k=1}^{n} \mu_{k}\left(\Delta_{k}\right)$. Note that when $a_{i}=\frac{1}{k}, i=1, \ldots, k$ and $\left|D_{n}\right|=n$ we get $\mu_{n}\left(\Delta_{n}\right)=\sigma P_{G_{n}}$, which is the situation in Theorem 4.

Now consider the plane tiled by one unit square tiles with sides parallel to the axis and center the coordinates $(m, n), m$ and $n$ integers. We color the tiles black or white and compute the measure the orbits of two groups of symmetries acting on the set of such tilings. For $m$ a a positive integer let $D_{m}=\{$ tiles with centers $( \pm m, k)$ or $(k, \pm m): k=-m,-m+1, \cdots, m-1, m\}$. Let $G_{n}=\prod_{k=1}^{2 n^{2}+1} z_{2}$ act on $D_{n^{2}}$ by interchanging tiles with central coordinates $\left( \pm n^{2}, k\right)$, $k=-n^{2}, \cdots, n^{2}$ and let $H_{n}=\prod_{k=1}^{2 n+1} \mathbf{Z}_{2}$ act on $D_{n^{2}}$ by interchanging tiles with central coordinates $\left( \pm n^{2}, k\right), k=-n, \cdots, n$. Now let $G=\prod_{n=1}^{\infty} G_{n}$ and $H=\prod_{n=1}^{\infty} H_{n}$. With $S=\{$ black, white $\}$, we define probability measures $\mu_{n}$ on $S^{D_{n}}$ by $\mu_{n}=\prod_{k=1}^{m_{n}} \gamma_{n}$, where $\nu_{n}($ black $)=\sqrt{\exp \left\{-\frac{1}{n(2 \sqrt{n}+1)}\right\}-\frac{\rho}{1}}+\frac{1}{2}$ and $\nu_{n}($ white $)=1-\nu_{n}($ black $)$. Let $\Delta\left(G_{n}\right)$ and $\Delta\left(H_{n}\right)$ be representatives for the orbits of $G_{n}$ and $H_{n}$ respectively on $S^{D^{2}}$ and let $\Delta(G)$ and $\Delta(H)$ be representatives for the orbits of $G$ and $H$ respectively on $S^{D}$. Then $\mu_{n}\left(\Delta\left(H_{n}\right)\right)=\exp \left(-1 / n^{2}\right)$ and so $\mu(\Delta(H))=\operatorname{limit}_{m \rightarrow \infty} \prod_{n=1}^{m} \mu_{n}\left(\Delta\left(H_{n}\right)\right)>0$. But $\mu_{n}\left(\Delta\left(G_{n}\right)\right)=\exp \left\{-\frac{2 n^{2}+1}{2 n^{3}+n^{2}}\right\}$ and so $\mu(\Delta(G))=\operatorname{limit}_{m \rightarrow \infty} \prod_{n=1}^{m} \mu_{n}\left(\Delta\left(G_{n}\right)\right)=0$.

\section{REFERENCES}

1. G. PÓLYA and R. C. READ, Combinatorial Enumeration of Groups, Graphs, and Chemical Compounds, Springer-Verlag, New York, 1987.

2. S. G. WILLIAMSON, Operator Theoretic Invariants and the Enumeration Theory of Pólya and de Bruijn, Journal of Combinatorial Theory 11 (1971), 122-138.

3. E. HEWETT and K. STROMBERG, Real and Abstract Analysis, Springer-Verlag, New York, 1965.

4. P. DIACONIS, Group Representations in Probability and Statistics , Institute of Mathematical Statistics, Hayward, California, 1988. 


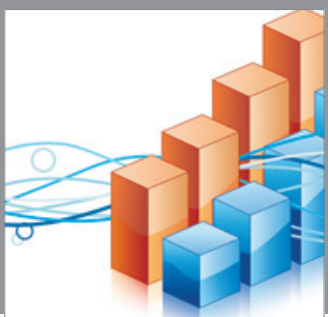

Advances in

Operations Research

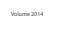

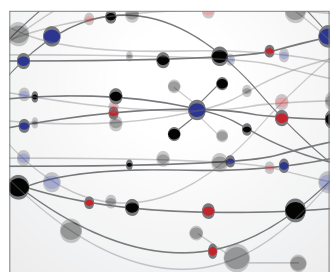

\section{The Scientific} World Journal
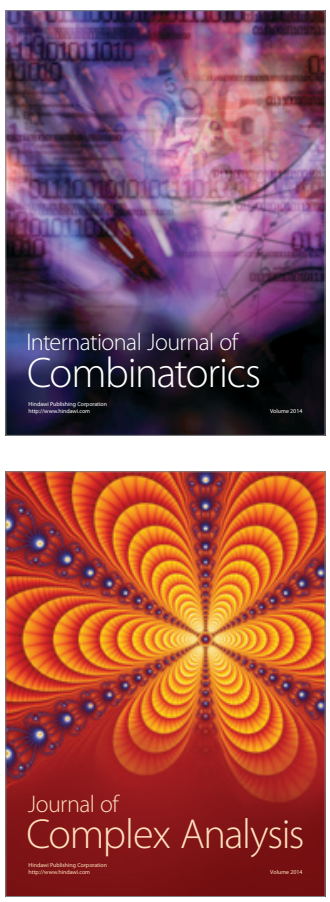

International Journal of

Mathematics and

Mathematical

Sciences
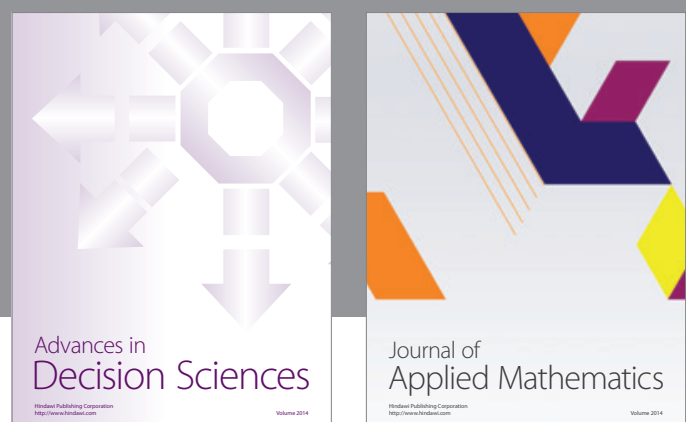

Journal of

Applied Mathematics
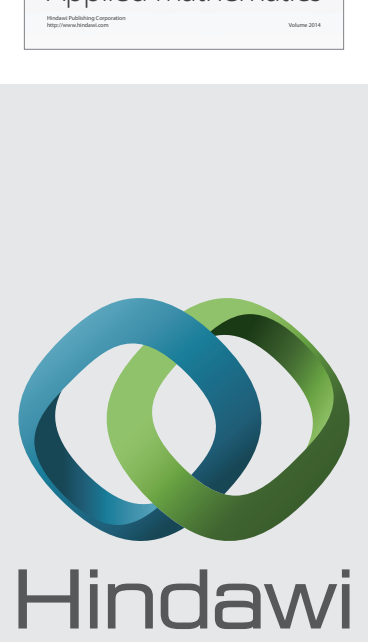

Submit your manuscripts at http://www.hindawi.com
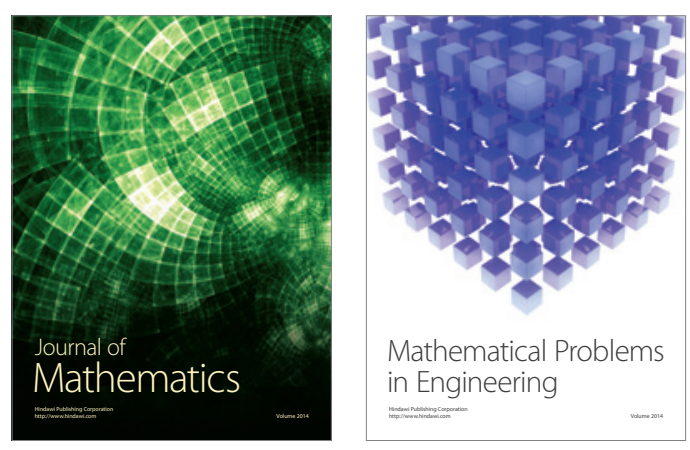

Mathematical Problems in Engineering
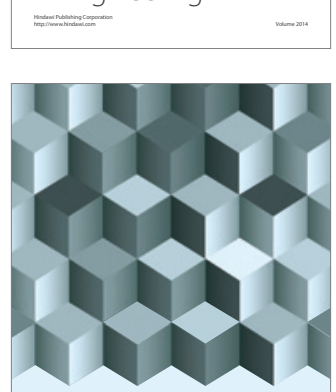

Journal of

Function Spaces
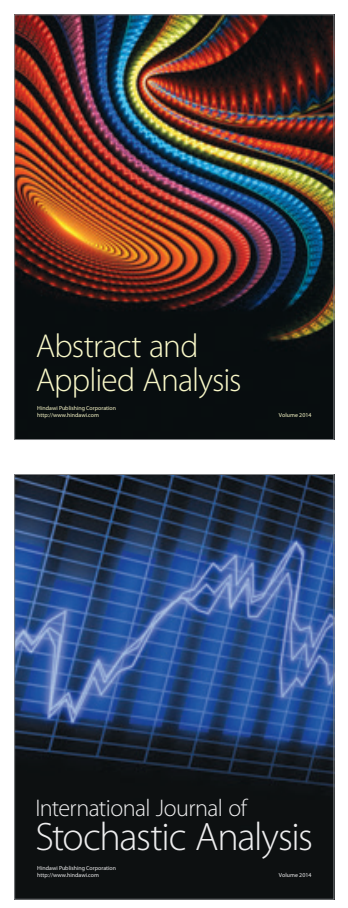

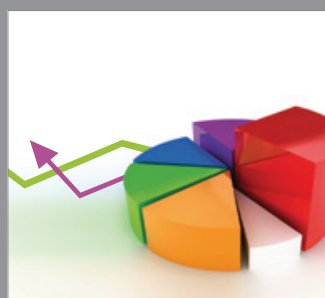

ournal of

Probability and Statistics

Promensencen
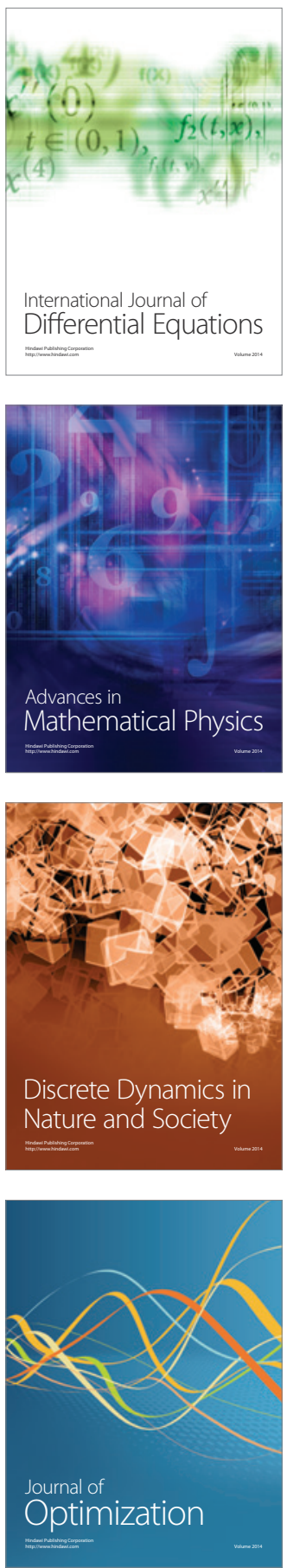